\title{
Dynamic Modeling of Process Technologies for Closed-Loop Water Recovery Systems.
}

Detailed chemical process simulations are a useful tool in designing and optimizing complex systems and architectures for human life support. Dynamic and steady-state models of these systems help contrast the interactions of various operating parameters and hardware designs, which become extremely useful in trade-study analyses. NASA's Exploration Life Support technology development project recently made use of such models to compliment a series of tests on different waste water distillation systems.

This paper presents dynamic simulations of chemical process for primary processor technologies including: the Cascade Distillation System (CDS), the Vapor Compression Distillation (VCD) system, the Wiped-Film Rotating Disk (WFRD), and post-distillation water polishing processes such as the Volatiles Removal Assembly (VRA) that were developed using the Aspen Custom Modeler and Aspen Plus process simulation tools. The results expand upon previous work for water recovery technology models and emphasize dynamic process modeling and results. The paper discusses system design, modeling details, and model results for each technology and presents some comparisons between the model results and available test data. Following these initial comparisons, some general conclusions and forward work are discussed. 